Meta

Journal des traducteurs

Translators' Journal

\title{
ZANolA, Maria Teresa (2018) : Che cos'è la terminologia [Qu'est-ce que la terminologie]. Rome : Carocci, 120 p.
}

\section{John Humbley}

Volume 64, numéro 2, août 2019

URI : https://id.erudit.org/iderudit/1068207ar

DOI : https://doi.org/10.7202/1068207ar

Aller au sommaire du numéro

\section{Éditeur(s)}

Les Presses de l’Université de Montréal

\section{ISSN}

0026-0452 (imprimé)

1492-1421 (numérique)

Découvrir la revue

Citer ce compte rendu

Humbley, J. (2019). Compte rendu de [Zanola, Maria Teresa (2018) : Che cos'è la terminologia [Qu'est-ce que la terminologie]. Rome : Carocci, 120 p.] Meta,

64(2), 561-562. https://doi.org/10.7202/1068207ar

Ce document est protégé par la loi sur le droit d'auteur. L'utilisation des services d’Érudit (y compris la reproduction) est assujettie à sa politique d'utilisation que vous pouvez consulter en ligne.

https://apropos.erudit.org/fr/usagers/politique-dutilisation/
Cet article est diffusé et préservé par Érudit.

Érudit est un consortium interuniversitaire sans but lucratif composé de l’Université de Montréal, l'Université Laval et l'Université du Québec à Montréal. Il a pour mission la promotion et la valorisation de la recherche. https://www.erudit.org/fr/ 


\section{DOCUMENTATION}

\section{Comptes rendus}

Zanola, Maria Teresa (2018): Che cos'è la terminologia [Qu'est-ce que la terminologie]. Rome: Carocci, 120 p.

Par rapport au français, à l'allemand, à l'espagnol et même à l'anglais, l'italien se démarquait jusqu'ici par l'absence d'introduction à la terminologie accessible à un public large ou semi-spécialisé. On se contentait du Pavel: didacticiel de terminologie en italien, cours en ligne qui propose aux débutants une toute première découverte, mais la terminologie italienne ne disposait encore d'aucun ouvrage de référence faisant la synthèse de ses théories et de ses pratiques, contrairement par exemple à la traduction spécialisée, dont la pédagogie s'appuie sur des manuels de qualité comme celui de Scarpa (2001). La lacune est désormais comblée, grâce à la parution du livre de Maria Teresa Zanola, qui propose à un public italophone une introduction à la fois tout à fait moderne et en même temps fermement ancrée dans la culture, dont le rôle fondateur est souvent passé sous silence. L'auteure, faut-il le rappeler, est idéalement placée pour concevoir une telle présentation. Elle a publié une étude remarquable sur l'émergence des terminologies dans le cadre de l'essor de l'encyclopédisme au dix-huitième siècle (Zanola 2014); elle a en outre été présidente du réseau de terminologie des langues latines, REALITER, et du Conseil européen des langues, sans oublier ses activités de base de professeure de terminologie et de communication spécialisée.

Matériellement, le livre ressemble à un numéro bien rempli de la collection française Que sais-je? et fait suite à celui d'Adamo et Della Valle (2017) sur la néologie. Il se distingue assez nettement de l'excellente introduction d'Alain Rey, publiée dès 1979 et révisée en 1992, au niveau de la bibliographie, dans la mesure où c'est la dimension encyclopédique qui prime ici, tandis que le lexicographe français mène surtout une profonde réflexion épistémologique.

Cette nouvelle publication est divisée en six chapitres, dont le contenu sera très brièvement évoqué ci-dessous. Nous nous permettrons ensuite quelques commentaires. Le premier chapitre s'ouvre sur la dimension culturelle de la terminologie, illustrée par les travaux des pionniers du dix-septième siècle italien qui réfléchissaient déjà sur la langue des arts et métiers. L'auteure passe ensuite au reste de l'Europe et à l'élaboration des nomenclatures (on pense à la botanique et au rôle de Linné) puis à celle des taxinomies (celle de la chimie avec Lavoisier et Guyton de Morveau étant l'exemple le plus frappant) avant d'examiner le rôle de l'encyclopédisme, déterminant pour une conceptualisation de la terminologie englobant sciences et techniques. Le ton est donné: la terminologie est l'élément de la langue qui permet la conception et la transmission de la culture - scientifique ou artisanale, d'où l'intérêt d'une théorie de la terminologie qui tienne explicitement compte de la dimension diachronique. Le deuxième chapitre aborde la disciplinarisation de la terminologie à partir des efforts de pionnier de Wüster et esquisse l'essentiel des nombreuses approches qui l'ont marquée depuis la fin du vingtième siècle, surtout au Canada, en France et en Espagne. L'intérêt de l'analyse diachronique en terminologie est rappelé, surtout dans le cadre de la création des dictionnaires et des encyclopédies. Le rôle de l'informatique est précisé, ainsi que quelques applications terminologiques des réalisations de l'équipe de Pise créée par le charismatique Antonio Zampolli. Ce chapitre se termine sur le rôle de la terminologie dans le cadre de la traduction spécialisée, auquel nous reviendrons dans les commentaires. Le troisième chapitre aborde les questions théoriques concernant d'abord la nature du terme, où l'analyse de Conceição (2005), fondée sur le rôle de la définition, sert de fil conducteur, pour aborder ensuite les relations entre terme et ontologie d'un côté (le travail de l'Institut Porphyre est cité en exemple) et terme et concept de l'autre. Le chapitre se termine sur des exemples concrets pris dans des projets de terminologie (REALITER en particulier), qui illustrent les conséquences pratiques et terminographiques des conséquences de décisions d'ordre théorique, en particulier concernant la rédaction de la définition. Le quatrième chapitre présente les principales activités terminologiques pratiquées dans le cadre de la traduction spécialisée, la documentation, la normalisation, avec, en corollaire, celles de l'extraction terminologique, leur typologie et les outils associés, pour finir par une description des banques de données. Une place particulière est accordée aux activités de l'association italienne de terminologie (Assiterm) ainsi qu'à REALITER. La dernière section de ce chapitre est consacrée à la formation universitaire en terminologie en relation avec les institutions italophones. C'est la terminographie qui fournit 
la matière du cinquième chapitre, sous la forme de l'analyse du projet de terminologie: les critères de sélection des sources et des termes en fonction des besoins, la conception et la confection de la fiche, la justification des différents champs. Le sixième et dernier chapitre («un réseau entre les connaissances») reprend les thématiques liées à l'organisation des connaissances évoquées dès l'introduction, pour aborder des questions importantes de politique linguistique. Partant du principe que les connaissances naissent dans les langues dans lesquelles elles prennent forme, l'auteure souligne l'intérêt pour la science de pratiquer une recherche plurilingue appuyée sur des terminologies fiables et également plurilingues.

Comme il se doit dans un manuel de type encyclopédique, l'auteure privilégie le socle commun des principes et des méthodes de la terminologie, désormais reconnue comme discipline. L'approche est donc consensuelle, ce qui n'empêche pas d'approfondir de nombreuses thématiques évoquées ni d'aborder les questions qui fâchent. Parmi ces dernières, citons un certain nombre de présupposés qui ne sont pas universellement acceptés, comme le pari que les connaissances se trouvent et peuvent être extraites des textes par le biais des termes. Quant aux points à approfondir, ils sont légion: c'est la fonction même d'une introduction d'aiguiller le débutant sur d'autres découvertes et de l'inviter à mener plus loin les réflexions amorcées au fil des pages. La question de la traduction revient dans tous les chapitres selon l'angle d'analyse retenu, c'est un des points forts du manuel. On aimerait poursuivre la discussion en s'interrogeant sur la traduction non pas des textes mais des termes. Le manuel nous indique comment identifier l'équivalent (ou les équivalents) d'un terme dans un texte dans les cadres de la traduction et de la terminographie. Mais que faire lorsque le terme posant problème est un néologisme dans les textes sources et que l'équivalent est introuvable? C'est une des questions que le lecteur se pose en lisant la section 2.5 , et le texte donne à réfléchir.

Résumons quelques qualités de ce livre. Constatons tout d'abord que son ambition encyclopédique se révèle parfaitement fondée: en l'espace de moins de 120 pages, l'auteure passe en revue l'essentiel des écrits et des réalisations en terminologie de ces cinquante dernières années. La bibliographie est riche, plurilingue (mais comportant d'importantes publications italiennes peu connues par ailleurs) et tout à fait à jour. C'est un livre qui est en prise directe avec les activités terminologiques internationales et leurs acteurs; il réserve une place importante aux nombreuses recommandations en terminologie en particulier de l'ISO. En même temps, l'étude est fermement ancrée dans la réalité italienne. Ce livre servira notamment à orienter le rôle de la terminologie dans le débat qui fait rage en Italie autour de la politique du «tout anglais» de certains milieux politiques et universitaires. C'est en effet un livre qui reconnaît l'intérêt de ne négliger ni la dimension diachronique ni les aspects culturels de la terminologie; c'est aussi un livre qui aborde directement la question de l'italien en tant que langue scientifique et technique.

JoHn Humbley

Université Paris Diderot, Paris, France Università di Verona, Vérone, Italie

\section{RÉFÉRENCES}

Adamo, Giovanni et Della Valle, Valeria (2017): Che cos'è un neologismo [Qu'est-ce qu'un néologisme]. Rome: Carocci.

ConCEIÇÃo, Manuel Célio (2005): Concepts, termes et reformulations. Lyon: Presses universitaires de Lyon.

Rey, Alain (1979/1992): La terminologie: noms et notions. Paris: Presses universitaires de France.

SCARPA, Federica (2001): La traduzione specializzata. Lingue speciali e mediazione lingüística [La traduction spécialisée. Langues de spécialité et médiation linguistique]. Milan: Ulrico Hoepli.

Zanola, Maria Teresa (2014): Arts et métiers au XVIII siècle - Études de terminologie diachronique. Paris: L'Harmattan.

Vidal Claramonte, Carmen África (2017): "Dile que le he escrito un blues", Del texto como partitura a la partitura como traducción en la literatura latinoamericana. Madrid: Iberoamericana/Vervuert, $186 \mathrm{p}$.

Pour l'entendre, il vous faut quitter la Plaza Mayor et prendre plutôt le passage piéton. Une fois vos pas posés dans les interstices de ce passage zébré, il surgit alors dans sa tenue bariolée, bigarrée, rapiécée. Tendez bien l'oreille: c'est Arlequin qui vous invite à parcourir avec lui l'essai de Carmen África Vidal Claramonte intitulé "Dile que le he escrito un blues", Del texto como partitura a la partitura como traducción en la literatura latinoamericana. Le patronage d'un tel personnage vous inquiète? Vous craignez que cet outsider par excellence, qui incarne le mélange, l'hybridité, voire l'incohérence, vous perde dans les dédales de la promenade proposée? Vous avez tort. Pour ouvrir la traduction à de nouveaux horizons conceptuels dans le contexte hybride de la mondialisation, pour l'aborder comme une activité hétérotopique à cheval sur des espaces et des temps épistémologiques variés, pour démontrer son rôle fondamental dans la transversalité des arts et leur 